\title{
Performance Analysis of FSO Link under Different Weather Conditions and Modulation Formats
}

\author{
Mehtab Singh \\ Assistant Professor, ECE Department \\ Satyam Institute of Engineering and Technology \\ Amritsar, India \\ mehtab91singh@gmail.com
}

\begin{abstract}
In the past few decades, Free Space Optics (FSO) has gained importance over the

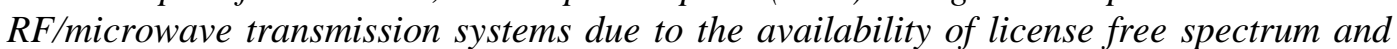
long range operations. Free Space Optical communication system has many advantages such as compact size, large bandwidth, lower cost and lease for implementation. Free Space Optical communication system also suffers from few limitations such as beam dispersion, atmospheric attenuation, and scintillation. One of the most significant limitations of FSO is the dependence of the performance of FSO system on the weather conditions. In this paper, a 10 Gbps FSO system has been designed and analyzed for different weather conditions and modulation formats at varying distances. From the results, it can be seen that as we move from clear weather conditions to heavy fog weather conditions, $Q$ factor of the received signal and maximum transmission distance both decreases. Also, NRZ modulation format performs better as compared to other modulation formats in an FSO communication link.
\end{abstract}

Keywords: Attenuation; beam dispersion; Free Space Optics; scintillations; weather conditions

\section{Introduction}

In Free Space Optical communication system, the information is transmitted from one place to another in the form of light traveling through the air in the same manner as in fiber optical communication system uses optical fiber cable. Free Space Optics has all the advantages of fiber optical communication system along with a lower cost of implementation and faster implementation speed [1]. Its numerous advantages include high data transfer speeds, lower cost, large bandwidth, fast installation, tight security and also license free long range frequency spectrum. Free Space Optics utilizes the principle of the laser-driven technology which utilizes light to carry information from one place to another in the air, same as in Fiber Optical Communication link, which utilizes light to carrying information from source to destination in an optical fiber. The main motivation behind Free Space Optics is to reduce the cost, time consumption and efforts in installation and implementation of fiber optical communication link and yet have high data transfer rates (10 Gbps and beyond) and large bandwidth to carry audio, video and voice signals. The implementation of Free Space Optic link is relatively simpler as compared to fiber optical communication link [2]. It consists of two systems each having an optical transceiver which has an optical transmitter and receiver to provide bidirectional data transfer capabilities. Each Free Space Optics system consists of an optical source having high power and a telescope which is used to transfer information in the form of light in the air which is then received by another telescope at the receiver end.

The receiving telescope consists of a highly sensitive receiver connected to it by an 
optical fiber. Free Space Optics technology can be utilized in numerous links such as satellite to ground, building to building, aircraft to ground and ship to ship. Free Space Optics has numerous advantages over traditional microwave and wireless communication system due to its ability to transmit data at high data rates with enhanced security and so on. In telecommunication systems, Free Space Optics is an optical communication technique to transfer data between two points in free space in the form of light. This technology comes useful where physical links between transmitter and receiver in the form of an optical fiber are not possible to implement [3]. In fiber optical communication system data is transferred between source and destination in the form of light pulses. In

Free Space Optics instead of using optical fiber to carry these light pulses, pulses are transmitted in the form of a narrow beam through the atmosphere. Light pulses containing information travels faster in the air as compared to within an optical fiber. Free Space Optics can be considered as an optical communication link with the speed of light. The performance of Free Space Optics link is dependent on weather conditions such as rain, dust, fog and storm. Free Space Optics is also considered for applications in military systems due to their enormous advantages. This paper investigates the performance of

Free Space Optics link under different weather conditions and modulation formats. Rest of the paper is organized as follows- System design and considerations are presented in Section 2. Results are presented and discussed in Section 3. The overall conclusion from this simulative investigation is given in Section 4.

\section{System Design and Considerations}

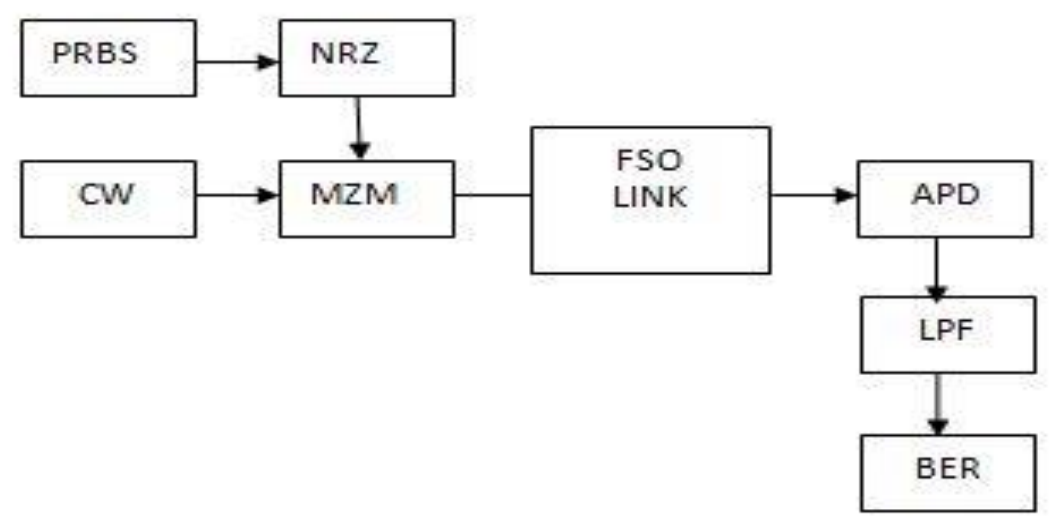

Figure 1. Free Space Optics Link

Figure No. 1 presents the basic design of Free Space Optic link and devices that have been utilized in this analytical work. The transmitter section consists of Pseudo-Random bit sequence (PRBS) generator, NRZ pulse generator, Mach-Zender modulator (MZM) and a continuous wave laser, whereas the receiver section consists of an APD photodiode and a low-pass Bessel filter. Also to analyze the performance of the communication link, BER analyzer has been used. Pseudo-Random bit generator generates information signal in the form of binary pulses i.e. 1010101 and so on. The binary pulses in the form of an electrical signal are directed toward NRZ pulse generator which converts these binary pulses to electrical signals. The output from NRZ pulse generator is directed towards Mach-Zender modulator whose other input end receives input from a continuous wave laser. The modulator converts the electrical signal to an optical signal. Now this optical signal travels through the atmosphere in the form of narrow beam electromagnetic wave and is received by a photo detector which converts this optical signal to its corresponding electrical signal. The attenuation of the free space medium between the transmitter and the 
receiver is taken to be $0.11 \mathrm{~dB} / \mathrm{Km}$ for clear weather condition, $5 \mathrm{~dB} / \mathrm{Km}$ for haze and 22 $\mathrm{dB} / \mathrm{Km}$ for fog [14].This electrical signal from photo detector is directed toward a low pass filter to remove any high-frequency noise present in the signal. The amount of error and the quality of the signal received can be analyzed using BER analyzer and power meter. Table.1 shows different simulation parameters used in this simulation.

Table 1. Simulation Parameters

\begin{tabular}{|c|c|}
\hline Parameter & Description/ Value \\
\hline Transmitting wavelength & $1550 \mathrm{~nm}$ \\
\hline Transmitting power & $4-8 \mathrm{~dB}$ \\
\hline Antenna diameter & $30 \mathrm{~cm}$ \\
\hline Link distance & $2000 \mathrm{~m}$ \\
\hline APD multiplication factor & 1 \\
\hline Data rate & $10 \mathrm{Gbps}$ \\
\hline APD quantum efficiency & 0.8 \\
\hline Filter type & Bessel Filter \\
\hline APD dark current & $1 \mu \mathrm{A}$ \\
\hline Divergence angle & $3 \mathrm{mrad}$ \\
\hline
\end{tabular}

\section{Results and Discussion}

In this paper, the performance of a Free Space Optical Communication Link is analyzed using different modulation formats and weather conditions. The performance of the FSO link is analyzed on the basis of Q Factor, total received power, and SNR of the received signal.

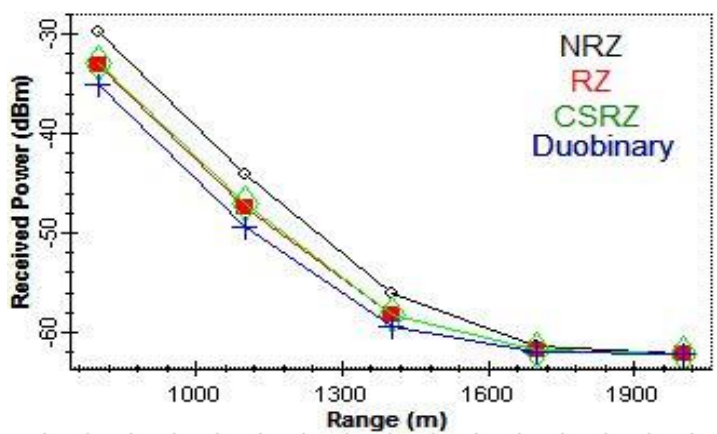

Figure 2. (a) Received Power v/s Range for Different Modulation Formats

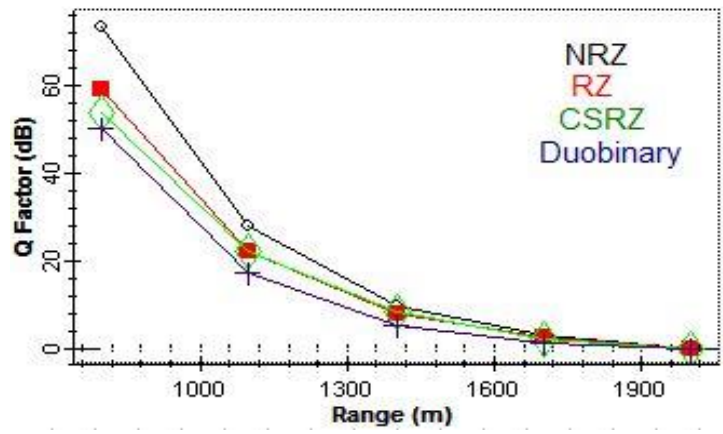

Figure 2. (b) Q Factor v/s Range for Different Modulation Formats 


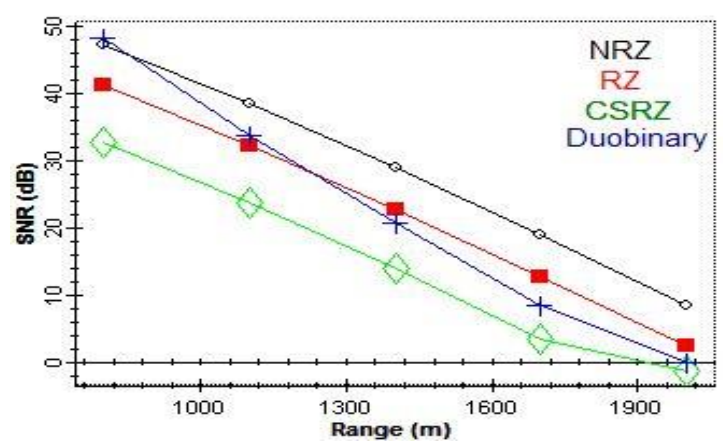

Figure 2. (c) SNR v/s Range for Different Modulation Formats

Figure 2(a), 2(b), and 2(c) indicate the graph between received power, Q Factor and SNR for varying link distance at different modulation formats. It can be observed from the results that there is a decrease in the received power which lies in the range $[-30,-32,-$ $33,-35] \mathrm{dBm}$ to $[-54,-56,-57,-60] \mathrm{dBm}$ for link distance of $1.5 \mathrm{Km}$ in case of NRZ, RZ, CSRZ and Duobinary modulation formats respectively and Q Factor values changes from $[77,66,55,50] \mathrm{dB}$ to $[12,11,10,8] \mathrm{dB}$ for link distance of $1.5 \mathrm{Km}$ in case of NRZ, RZ, CSRZ and Duobinary modulation format respectively and SNR ratio lies in the range [48, $42,47,32] \mathrm{dB}$ to $[30,25,20,17] \mathrm{dB}$ for link distance of $1.5 \mathrm{Km}$ in case of NRZ, RZ, CSRZ and Duobinary modulation format respectively.



Figure 3. (a) Received Power v/s Range for Different Weather Conditions

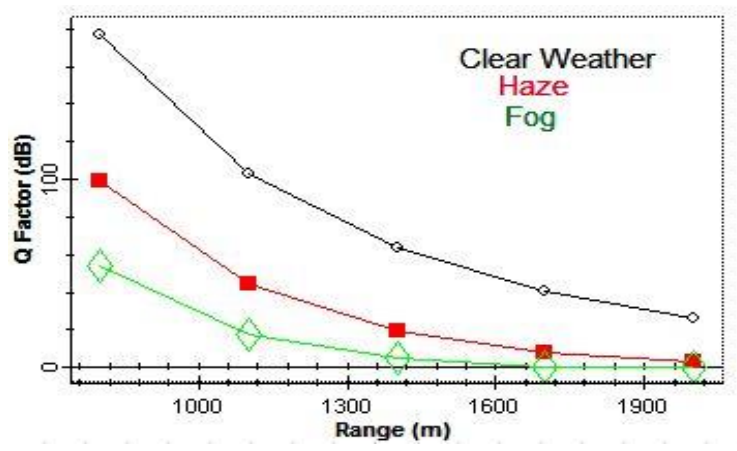

Figure 3. (b) $Q$ Factor v/s Range for Different Weather Conditions 


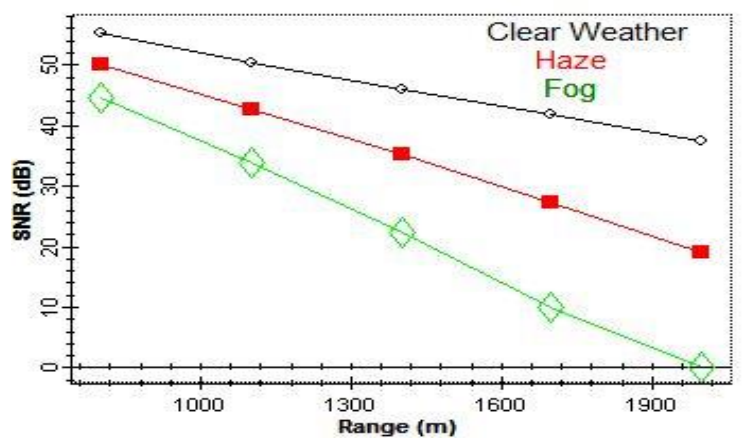

Figure 3. (c) SNR v/s Range for Different Weather Conditions

Figure 3(a), 3(b), and 3(c) indicates the graph between the received power, Q Factor and SNR for varying link distance at different weather conditions. It can be observed from the results that there is a decrease in the received power which lies in the range $[-15,-27,-$ $33] \mathrm{dBm}$ to $[-30,-47,-62] \mathrm{dBm}$ for link distance of $1.5 \mathrm{Km}$ in case of clear weather condition, haze and fog respectively and Q Factor values changes from $[120,100,80] \mathrm{dB}$ to $[80,50,33] \mathrm{dB}$ for link distance of $1.5 \mathrm{Km}$ in case of clear weather condition, haze and fog respectively and SNR ratio lies in the range $[55,50,44] \mathrm{dB}$ to $[50,40,20] \mathrm{dB}$ for link distance of $1.5 \mathrm{Km}$ in case of clear weather condition, haze and fog respectively.

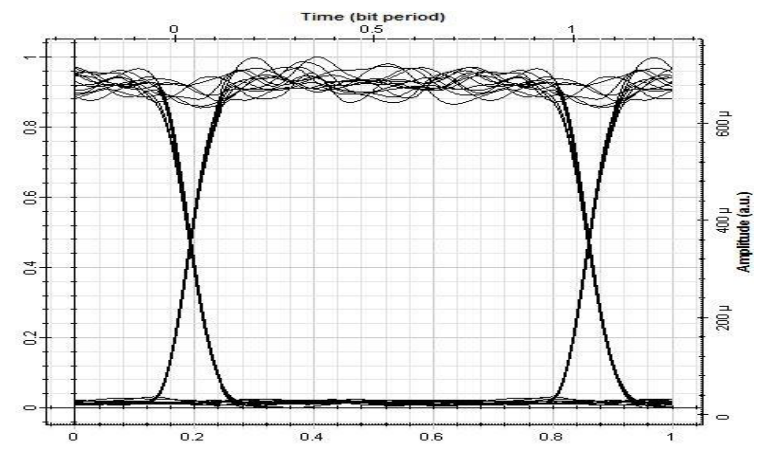

Figure 4. (a) Eye Diagram of the Received Signal Under Clear Weather Condition

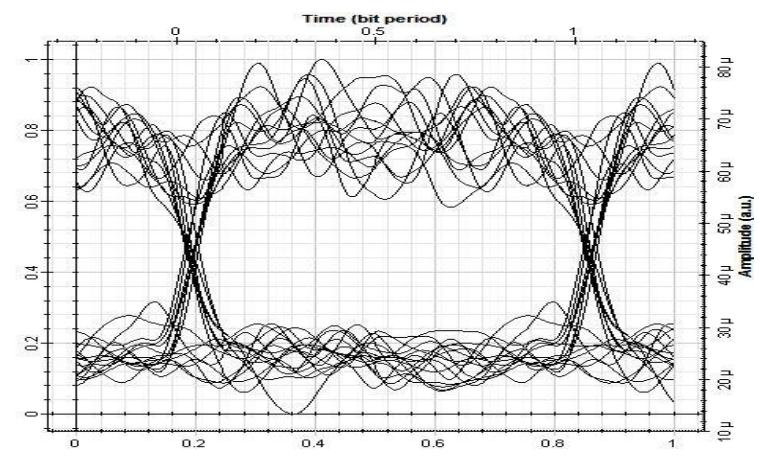

Figure 4. (b) Eye Diagram of the Received Signal Under Haze Weather 


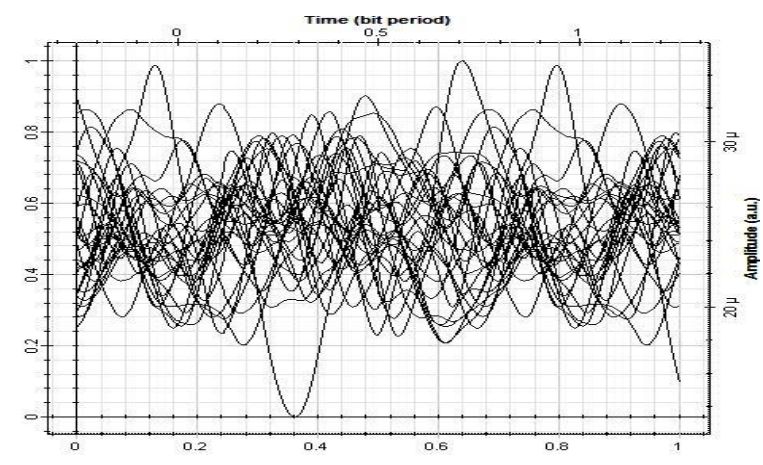

Figure 4. (c) Eye Diagram of the Received Signal Under Fog Conditions

Figure 4 (a), 4(b) and 4 (c) indicates the eye diagrams of the received signal at a link distance of $1.5 \mathrm{Km}$ in FSO communication link for clear weather condition, haze and fog weather condition respectively. In this paper, the impact of using EDFA as a pre-amplifier in order to improve the performance of the FSO link has also been simulated and discussed.

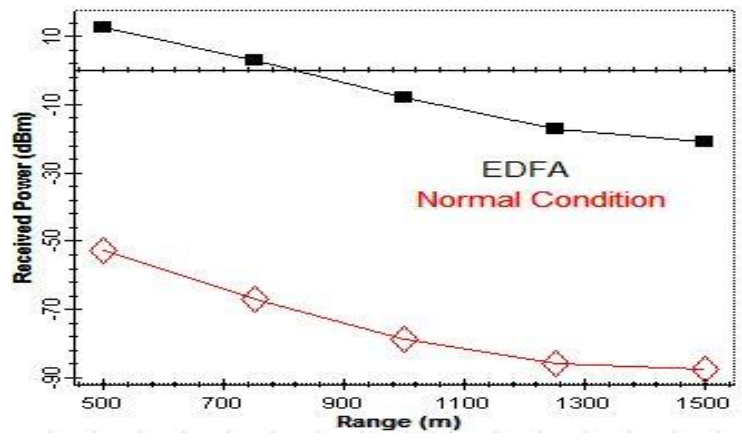

Figure 5. (a) Received Power v/s Range for Different Systems



Figure 5. (b) SNR v/s Range for Different Systems

Figure 5 (a) and 5(b) indicates the total received power and SNR for varying link distance in an FSO link for different systems. It can be seen that the total received power reduces from $10 \mathrm{dBm}$ to $-13 \mathrm{dBm}$ for a link distance of $500-1550 \mathrm{~m}$ in the case when EDFA is used in the communication link, on the other hand, received power reduces from $-50 \mathrm{dBm}$ to $-80 \mathrm{dBm}$ for a link distance of $500-1550 \mathrm{~m}$ in the case when EDFA is not used in the communication link. Also, SNR of the received signal reduces from $50 \mathrm{~dB}$ to $20 \mathrm{~dB}$ for a link distance of $500-1500 \mathrm{~m}$ in the case when EDFA is used in the system and SNR reduces from $37 \mathrm{~dB}$ to $0 \mathrm{~dB}$ for a link distance of $500-1550 \mathrm{~m}$ in the case when EDFA is not used in the communication link. Fig. 6(a) and 6(b) indicate the eye diagrams 
of the received signals for different systems under clear weather conditions in an FSO link at $1550 \mathrm{~m}$ link distance.

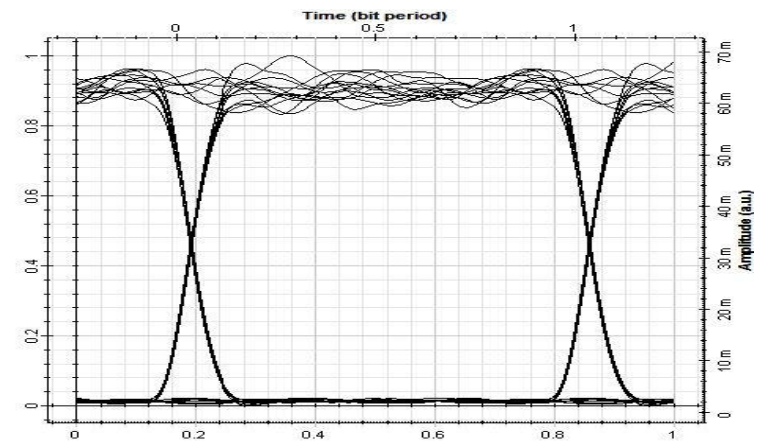

Figure 6. (a) Eye Diagram of the Received Signal Under Clear Weather with EDFA

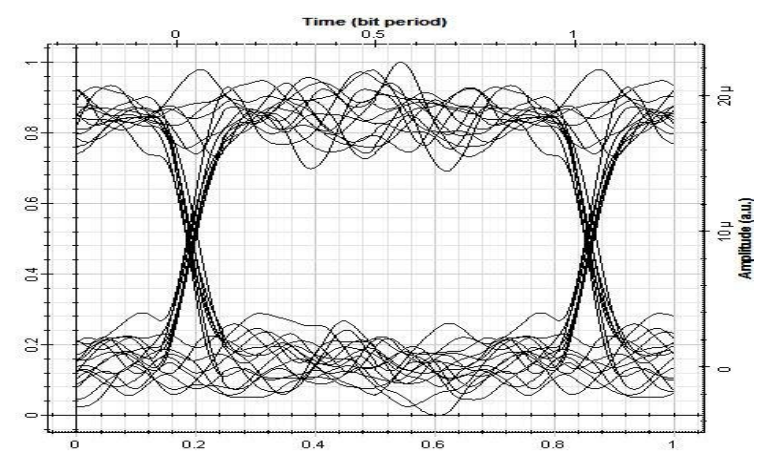

Figure 6. (b) Eye Diagram of the Received Signal Under Clear Weather Without EDFA

\section{Conclusion}

In this paper, the impact of different modulation formats and weather conditions on the performance of Free Space Optical Communication system has been analyzed and discussed. Performance has been analyzed on the basis of Q Factor, SNR, and total power of the received signal. From the results, it can be observed that $\mathrm{Q}$ Factor lies in the range 77 to $12 \mathrm{~dB}$ and total received power lies in the range -30 to $-54 \mathrm{dBm}$ and SNR lies in the range 48 to $30 \mathrm{~dB}$ for NRZ modulation format and link distance of $1.5 \mathrm{Km}$. From the results presented and discussed, it can be concluded that NRZ modulation format performs better as compared to RZ, CSRZ, and Duobinary modulation formats in an FSO communication link. Also, Q Factor lies in the range 120 to $80 \mathrm{~dB}$ and total received power lies in the range -15 to $-30 \mathrm{dBm}$ and SNR lies in the range 55 to $50 \mathrm{~dB}$ for clear weather condition. Under clear weather condition, the FSO link prolongs to $2 \mathrm{Km}$, whereas for haze and fog conditions the link prolongs to $1.3 \mathrm{Km}$ and $950 \mathrm{~m}$, respectively. Also, from the results presented it can be concluded that the performance of FSO communication link can be enhanced by using amplifiers which enhance system performance under different weather conditions. 


\section{References}

[1] H. Henniger and O. Wilfert, "An Introducttion to Free Space Optical Communications. Radioengineering, vol. 19 , (2010) June.

[2] B. Barua, "Comparison the Performance of FreeSpace Optical Communication with OOK and BPSK Modulation under Atmospheric Turbulence", International Journal of Engineering Science and Technology (IJEST), vol. 3, (2011) May.

[3] W. O. Popoola, "BPSK Subcarrier Intensity Modulated Free-Space Optical Communications in Atmospheric Turbulence. JOURNAL OF LIGHTWAVE TECHNOLOGY, vol. 27, (2009) April 15.

[4] L. Nadeem, E. Awan, "M. S. 2009. Optical Wavelengths Comparison for Different Weather Conditions.",IEEE, (2009).

[5] B. Epple, "Simplified Channel Model for Simulation of Free-Space Optical Communications", vol. 2, (2010) May.

[6] K. Kiaseleh, "Performance of Coherent DPSK Free Space Optical Communication System in Distributed Turbulence", IEEE Transactions Communication, vol. 54, (2006) April 4.

[7] "Info-Communications Development Authority (IDA) of Singapore", 2002. A Trial-Based Study of Free-Space Optics Systems in Singapore, (2002) October.

[8] I. I. Kim, B. McArthur and E. Korevaar, "Comparison of Laser Beam Propagation at 785nm and 1550nm in Fog and Haze for Optical Wireless Communications", SPIE Proceedings, vol. 4214, pp.2637.

[9] H. Nistazakis, T. A Tsiftsis and G. S. Tombras, "Performance Analysis of Free Space Optical Communication Systems over Atmospheric Turbulence Channels", IET Communication, vol. 3, no. 8, pp.1402-1408.

[10] H. Manor and S. Arnon, "Performance of an Optical Wireless Communication System as a Function of Wavelength", Applied Optics, vol. 42, no. 21, pp. 4285-4293.

[11] A. S. Mohd Supa'at, A. B Mohammad, Y. T. Tong and S. M. Idrus, "Unguided Optical Communication: Design a Mndewa”, S. M., Huang, D., Yuan, X. 2008. A Survey of Atmospheric Turbulence on Laser Propagation. Asian Journal of Information Technology, vol. 7, no. 7, pp. 307-312.

[12] S. M. Mndewa, D. Huang and X. Yuan, "A Survey of Atmospheric Turbulence on Laser Propagation", Asian Journal of Information Technology, vol. 7, no. 7, pp. 307-312.

[13] B. S Naimullah, M. Othman, A. K Rahman, S.I. Sulaiman, S. Ishak, S. Hitam and S. A Aljunid, "Comparison of Wavelength Propagation for Free Space Optical Communications", International Conference of Electronic Design, pp. 1-5.

[14] I. I Kim, R Stieger. J. A Koontz. C. Moursund, M. Barclay, P. Adhikari, J. Schuster and E. Korevaaret, "Wireless Optical Transmission of Fast Ethernet", FDDI, ATM and ESCON Protocol Data Using the TerraLink Laser C.

[15] O. Bouchet, H. Sizun, C. Boisrobert, F. de Fornel and P.-N. Favennec, "FreeSpace Optics:Propagation and Communication" Publisher Hermes Science in France, 2004nd Evaluation in Malaysia Atmosphere, pp. 269-271. 